\title{
A Novel Human Parsing Method Enhanced by Cross-Refinement
}

\author{
Benzhu Xu ${ }^{1,2}$, Qing Han $^{2}$, Liping Zheng ${ }^{1,2}$ and Gaofeng Zhang ${ }^{1,3+}$ \\ ${ }^{1}$ School of Software, Hefei University of Technology, Hefei 230009, China \\ ${ }^{2}$ School of Computer and Information, Hefei University of Technology, Hefei 230009, China \\ ${ }^{3}$ Anhui Province Key Laboratory of Industry Safety and Emergency Technology, Hefei, China
}

\begin{abstract}
The existing human parsing methods lead to some semantic errors and incomplete semantic recognition. To solve this two problems, we design a novel approach, called Cross Refinement Network (CRN). It is built based on the Part Grouping Network (PGN). The CRN remains the same as the PGN in the backbone network and the final refinement block, and uses the ResNet-101 and the Refinement branch separately, just like the PGN. Compared to the PGN, CRN innovatively utilizes the atrous convolution to map the last three feature modules of the backbone network into the feature space, and proposes the cross-refinement module, which consists of the semantic segmentation refinement branch and the edge detection refinement branch, to cross-merge the output feature maps. Experiments are performed based on Crowd Instance-level Human Parsing(CIHP), Pascal-Person-Part (PPP), and LIP datasets separately. The results show that our method outperforms the typical methods on the task of human parsing.
\end{abstract}

Keywords: Semantic Segmentation, Human Parsing, Part Grouping Network, Cross Refinement Network

\section{Introduction}

In recent years, methods based on deep neural network have made great progress in semantic segmentation task. In 2014, Long etc. [1] conducted end-to-end segmentation of natural images with Fully Convolutional Networks (FCNs) for the first time. L. C. Chen etc. [2] introduce atrous convolution to explicitly control the resolution of feature responses computed by Deep Convolutional Neural Networks. In [3], they employ atrous convolution with different sampling rates in parallel to capture multi-scales context. These semantic segmentation methods promote the accuracy improvement of the human parsing task. Human parsing is generally regarded as a fine-grained semantic segmentation, it aims at assigning a semantic category to each image pixel of the human body. Solving this problem is critical in several application scenarios including human analysis, virtual try-on and video surveillance [4].

Differs from semantic segmentation task which needs only to assign all the pixels belonging to one instance to the same semantic label, such as car, horse, person, the human parsing task requires to distinguish those semantic parts of one person, such as head, torso, arms and legs. Compared to the former, the human parsing task is even more challenging.

However, the current human parsing methods usually has the two problems: 1) generate some semantic errors (i.e. the semantic label of skirt is recognized as pants), 2) incomplete semantic recognition (absence of semantic information of head).

To solve these two problems, we introduce a novel architecture named Cross Refinement Network (CRN), which is built based on the PGN [5]. The whole CRN pipeline can be divided into three sub-modules, i.e. the shared backbone network, the cross refinement module and the final refinement branch. In particular, the cross refinement module we proposed aims to merge the extracted feature map into the feature space

\footnotetext{
${ }^{+}$Corresponding author. Tel.: +86-551-63831881; fax: +86-551-63831870.

E-mail address: g.zhang@hfut.edu.cn.
} 
alternately for the joint refinement of semantic segmentation and edge detection. Specifically, after obtaining the shared weights through ResNet-101 same as PGN, we adds convolution operation to map the last three modules of ResNet-101 into semantic feature space and edge feature space to obtain the corresponding extracted feature maps, attaches semantic segmentation refinement branch and the edge detection refinement branch respectively to fuse the above extracted features map alternately. Experiments on the publicly available datasets shows that our method improved the effectiveness of human parsing compared to the typical method to a certain extent.

We will introduce semantic segmentation, human parsing, and the PGN architecture in Section 2. Our method will be elaborated in Section 3. Our experimental results and conclusion will be shown in Sections 4 and 5 separately.

\section{Related work}

\subsection{Semantic Segmentation}

Semantic segmentation aims to assign each pixel with a semantic categories, such as car, horse, person. It is one of the most relevant research directions to human parsing. Since the FCNs [1] on semantic segmentation task was proposed in 2014, some newer and better ones based on the FCNs have been derived.

Specifically, N. Souly etc. [6] proposes a semi-supervised framework based on generative adversarial networks, which can add large fake visual data to force real samples to be close in the feature space. O. Ronneberger etc. [7] raises U-Net architecture, which consists of a contracting path to capture context and a symmetric expanding path that enables precise localization. It can be trained end-to-end with very few images, and outperforms the prior best method. V. Badrnarayanan [8] proposes the SegNet, which consists of an encoder network and a corresponding decoder network followed by a pixel-wise classification layer. It reduces trainable parameters compared to the other competing architectures efficiently. H. Zhao etc. [9] proposes Pyramid Scene Parsing network (PSPNet) for solving pixel-level prediction tasks, which aims to extract feature information of different regions through the pyramid pooling module and fuse them to obtain global contextual information.

Besides, there are some other classic structure such as VGG [10] which uses the combinations of multiple small filters instead of large filters. It can deepen the depth of the network under the condition of the same receptive field. K. He etc. proposes ResNet [11] which contains 152 neural network layers and is trained successfully with residual unit, and verified the effectiveness of the residual unit in deep network model. F. Yu etc. [12] proposes a novel architecture with dilated convolution module for dense prediction tasks, which can aggregate multiscale contextual information systematically without increasing the amount of parameters and calculations as well as ensuring the resolution of feature space.

These methods above outperform on semantic segmentation task. Guided by this intuition, we try to tackle human parsing problem via the novel CRN architecture which utilizes the improved ResNet, termed Deeplab-v2 [2], the atrous convolution [12] and the Pyramid Pooling structure [9].

\subsection{Human Parsing}

Human parsing aims at assigning a semantic category to each image pixel of the human body. Solving the problem is the key to promoting human-centered analysis and research. According to different scenarios, it can be divided into single-person parsing and multiple-person parsing or instance-level human parsing.

Luo etc. [13] uses Macro-Micro Adversarial Network (MMAN) to optimize the local discontinuity and semantic discontinuity existed in the results of human parsing, and achieves relatively good performance compared with other methods on LIP and PPP dataset. K. Cong etc. [14] develops a self-supervised sensitive learning model based on deep convolutional neural network, which forcing the results of segmentation to be semantically consistent with the structure of human joints. Excellent parsing results can be obtained on the single-person dataset by using this method, but the effect is not good on the multiple-person dataset. However, The PGN architecture he put forward in [5] can solve the problem of multiple-person parsing very well. It employs the instance-aware edge information to group semantic parts into distinct person instances. 
The human parsing methods above generate some semantic errors (i.e. the semantic label of skirt is recognized as pants) and incomplete semantic recognition (absence of semantic information of head). To solve these two problems, we propose the CRN which is built based on the PGN. In particular, we involve the cross refinement module which consists of the semantic segmentation refinement branch and the edge detection refinement branch, to cross-merge the output feature map. Experimental results show that our method can gain more accurate semantic segmentation results on the human parsing task.

\subsection{PGN}

The PGN [5] architecture extract shared feature map information through a unified backbone network firstly. Then, it attaches semantic part segmentation and instance-aware edge detection. Technically, both of the sub-tasks are pixel-wise classification problems. Due to the superiority of ResNet-101 in dense forecasting tasks, it is used as the backbone network of the PGN architecture. They add two parallel branches to semantic part segmentation and instance-aware boundary detection respectively. To explore and employ the semantic relevance of these two tasks, they further introduce refinement branch which benefit the two targets by using complementary contextual information. PGN performs well on the multiple-person parsing tasks, but still exist the problem of lacking semantic information, such as: segmentation of hand ,foot and head is not very good. Therefore, we propose the Cross Refinement Network for this problem, which can solve the problem of lacking semantic information.

\section{Cross Refinement Network}

In this section, we will introduce our method in three steps. Firstly, We illustrate the CRN systematically in Section 3.1, then, the backbone network and refinement module, which are the same as PGN, are introduced by Section 3.2, and finally the innovative part of the method is proposed in Section 3.3.

\subsection{CRN Architecture}

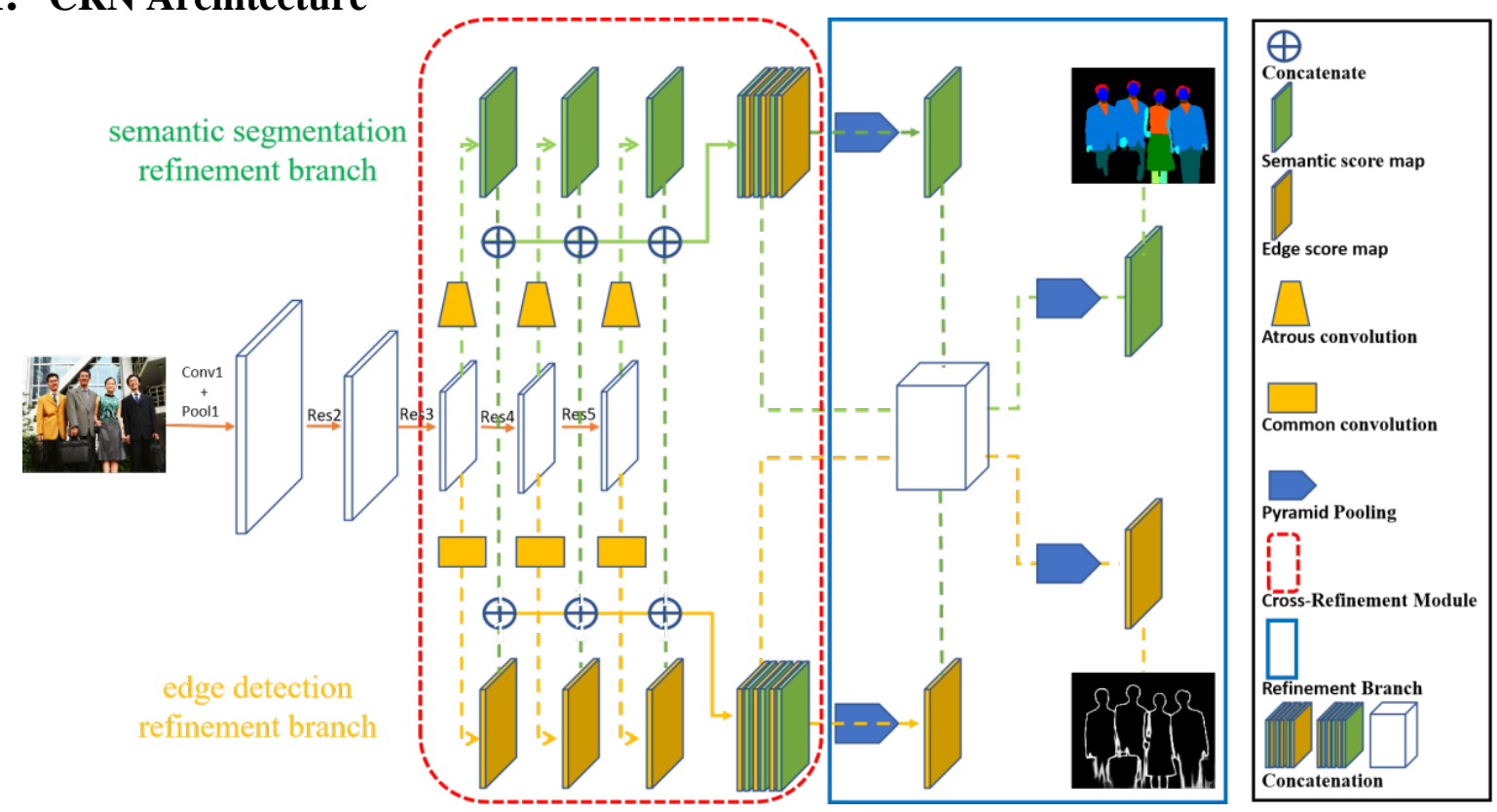

Fig. 1: Illustration of the CRN Pipeline

In this section, we present a general pipeline of the CRN in Figure 1, and detail each part of the architecture. Firstly, similar to the PGN structure, we use ResNet-101 as the unified network to train and optimize the shared weights jointly. Secondly, we involve the cross-refinement module consists of the semantic segmentation refinement branch and the edge detection refinement branch, to obtain the complementary contextual information (see the red dotted box in Figure 1). Finally, we employ a refinement branch to make the two sub-tasks further benefit each other (see the blue rectangle box in Figure 1).

Specifically, for a given input image, we use ResNet-101 to extract the shared feature information, and map the last three feature modules of ResNet-101 into the semantic feature space and the edge feature space separately by adding atrous convolution and common convolution. Then, we append the novel 
cross-refinement module which will be introduced in Subsection 3.3, which consists of the semantic segmentation refinement branch and the edge detection refinement branch. We obtain the semantic concatenated feature map and the edge concatenated feature map by cross-merging the extracted semantic feature map and extracted edge feature map obtained by the convolution operation. Finally, we obtain the semantic score map and the edge score map through further refinement.

\subsection{Shared Backbone Network and Refinement branch}

Our method keeps some parts of PGN, including the Shared Backbone Network and the Refinement branch. Specifically, we use ResNet-101 with atrous convolution, Deeplab-v2[2] as our human feature encoder, which performs well in dense prediction tasks. It uses atrous convolution which can expand the receptive field while keeping the resolution of feature space unchanged. This structure can avoid the risk of losing context information caused by the use of down-sampling.

Refinement branch combines the remapped feature maps through the pyramid pooling modules with the concatenate feature maps from both the semantic segmentation refinement branch and edge detection refinement branch. Then, it further optimize segmentation and edge results mutually through two another pyramid pooling modules.

\subsection{Cross Refinement Module}

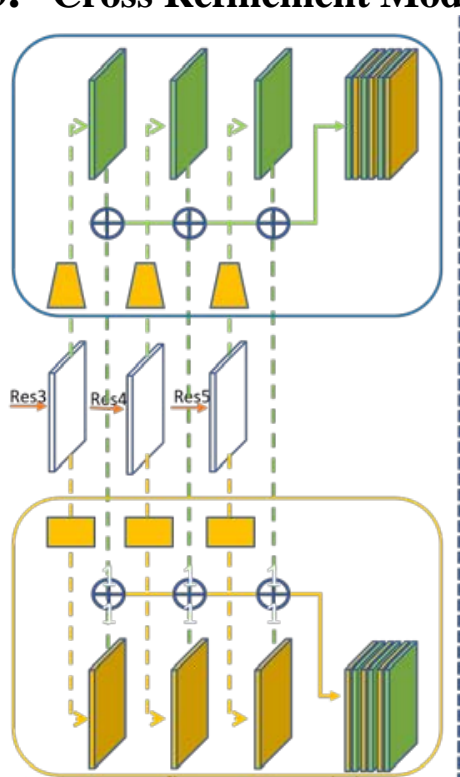

(a) cross-refinement module

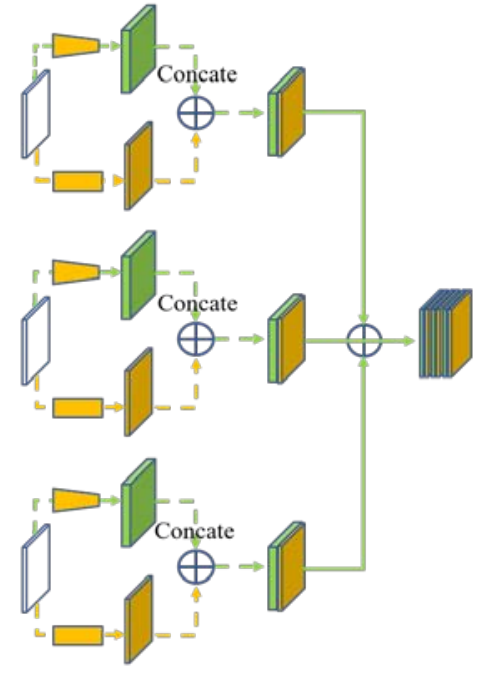

(b) semantic segmentation refinement branch

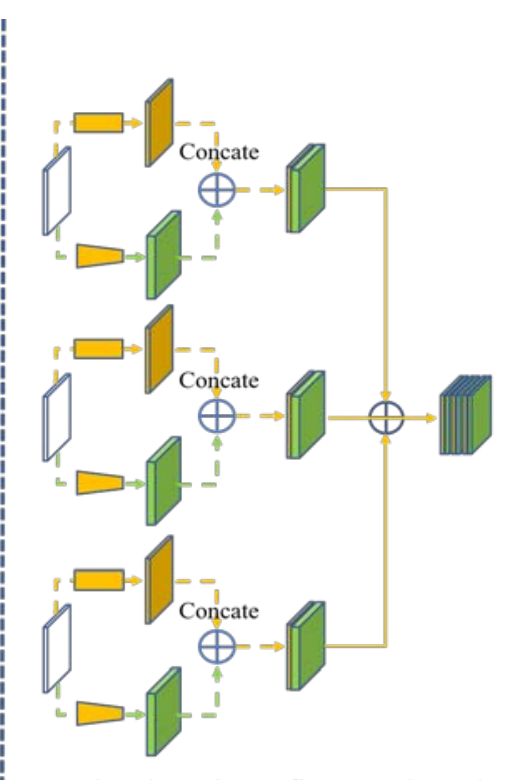

(c) edge detection refinement branch

Fig. 2: cross-refinement module and the two branches

Inspired by the Refinement branch of PGN, we design a novel cross-refinement structure, as shown in Figure 2. (a) corresponds red dotted box in Figure 1, (b) and (c) are the detailed descriptions of the two branches of the cross-refinement module, where the semantic segmentation refinement branch in (b) corresponds to the blue box in (a), and the edge detection refinement branch in (c) corresponds to the orange box in (a).

The atrous convolution with different sampling rates and the common convolution are innovatively employed to map the last three feature modules of ResNet-101 to the semantic feature space and the boundary feature space separately. We do this for the good performance of the atrous convolution in dense feature extraction. It can not only expand the receptive field while keeping the resolution of feature space unchanged, but also avoid the risk of losing context information caused by the use of down-sampling. For the edge detection part of the character image, because of it contains little context information, we can use the common convolution to achieve the extraction of boundary information.

The cross-refinement module we proposed innovatively consists of the semantic segmentation refinement branch and the edge detection refinement branch. This structure merges the extracted feature map into the feature space alternately for the joint refinement of the semantic segmentation and the edge detection. In Figure 2.a, we add the extracted boundary feature onto the semantic feature space to fuse the information. Correspondingly, we attach the extracted semantic feature onto the boundary feature space in Figure 2 (b). In the last three modules of ResNet-101, we use the same mechanism to get the corresponding three feature structure, and fuse them again to get the semantic concatenate feature map and boundary concatenate feature 
map.

Compared to PGN, We regard that the two branches of CRN are highly correlated with each other by the shared feature maps information. The cross-refinement module of our CRN further refine the boundary information and the semantic segmentation to make the two branches mutually benefit from each other by employing complementary contextual information. It can achieve more accurate parsing and improve pixel accuracy in human parsing.

\section{Experiment}

In this section, we firstly introduce the implementation details of our method, metrics and datasets. Then, we perform a comprehensive comparison of our CRN with some typical existing methods, on the basis of CIHP [5], PPP [15] and LIP [14].

\subsection{Experiment Preparation}

(1) Implementation Details

Following the previous works, we employ the ResNet-101 [3] that is pre-trained on the Pascal VOC dataset [16] as the backbone network. We set the input $512 * 512$ by cropping from the images randomly, and initialize the learning rate to 0.00001. semantic segmentation part loss weight a is set to 50, and edge detection loss weight $\beta$ is set to 0.005 . The data are enhanced by random scaling, random cropping, and random rotation and left-to-right flipping of the training image. The experimental environment required is set up on the server with Centos7.6 as the operating system and NVIDIA Tesla M60 as the graphics card.

(2) Metrics

We employ three standard metrics for the human parsing task, including mean intersection over union (mIoU) [13], mean accuracy, and recall. Among them, the mIoU metric generally reflect the parsing performance of the semantic segmentation methods.

$$
\begin{aligned}
& \text { Io } U=\frac{T P}{T P+F P+F N} \\
& \text { Accuracy }=\frac{T P+T N}{T P+T N+F P+F N} \\
& \text { Recall }=\frac{T P}{T P+T N}
\end{aligned}
$$

Equations (1-3) are the calculation formulas of IoU, Accuracy and Recall respectively. Among them, TP indicates that predict correctly, the prediction result is positive, and the truth is positive; FP indicates that predict wrongly, the prediction result is positive, and the truth is negative; FN indicates predict wrongly, the prediction is negative, and the truth is positive; TN indicates predict correctly, the prediction is negative, and the truth is negative.

(3) Datasets

We list the public available datasets used for human parsing in Table 1. PPP [13] included in Pascal VOC2010 [16], which contains 1716 train images,1817 test images. The ground truth label consists of six semantic parts including head, torso, upper/lower arms, upper/lower legs and one background class. CIHP [5] is the largest multiple-person human parsing dataset to date, which contains 38280 diverse character images with pixel-level annotations on 20 categories and instance-level identification. LIP [14] is the largest human parsing dataset, which contains 50462 images with 19 semantic human part labels at the pixel-level.

Table 1: Comparison of datasets used in the experiment for human parsing

\begin{tabular}{ccccccc}
\hline datasets & Persons/image & Total set & Train set & Validation set & Test set & Categories \\
\hline PPP [13] & 2.2 & 3533 & 1716 & - & 1817 & 7 \\
CIHP [9] & 3.4 & 38280 & 28280 & 5000 & 5000 & 20 \\
LIP [11] & 1 & 50462 & 30462 & 10000 & 10000 & 20 \\
\hline
\end{tabular}

\subsection{Comparisons of Human Parsing}

(1) Evaluation on the PPP dataset

We compare the performance of our CRN with the typical methods on the PPP dataset, and show the comparison results of human parsing with three baseline methods [13,2,5] in Table 2. Our CRN method outperforms all baselines in the terms of all the three metrics, and compared with the best semantic segmentation methods, it achieves $1.11 \%$ higher in mIoU values. This performance improvement confirms the effectiveness of the CRN we proposed. 
Table 2: Comparison results with the current methods on PPP dataset

\begin{tabular}{cccc}
\hline Method & MeanIoU(\%) & Mean ACC(\%) & Recall(\%) \\
\hline MMAN [13] & 58.45 & 88.27 & 91.85 \\
DeepLab-v2 [2] & 64.94 & 86.91 & 96.07 \\
PGN [5] & 68.40 & 93.19 & 90.97 \\
\hline CRN(Ours) & $\mathbf{6 9 . 5 1}$ & $\mathbf{9 3 . 9 4}$ & $\mathbf{9 6 . 3 1}$
\end{tabular}

As shown in Figure 3. The first two columns are the original images and the Ground Truth, the next four columns are the human parsing map by MMAN, Deeplab-v2, PGN and the CRN respectively. It can be observed that compared with the other methods, CRN can achieve even more accurate parsing results emphasized with white boxes in Figure 3, which confirms the effectiveness of our method in intensive character image parsing tasks.
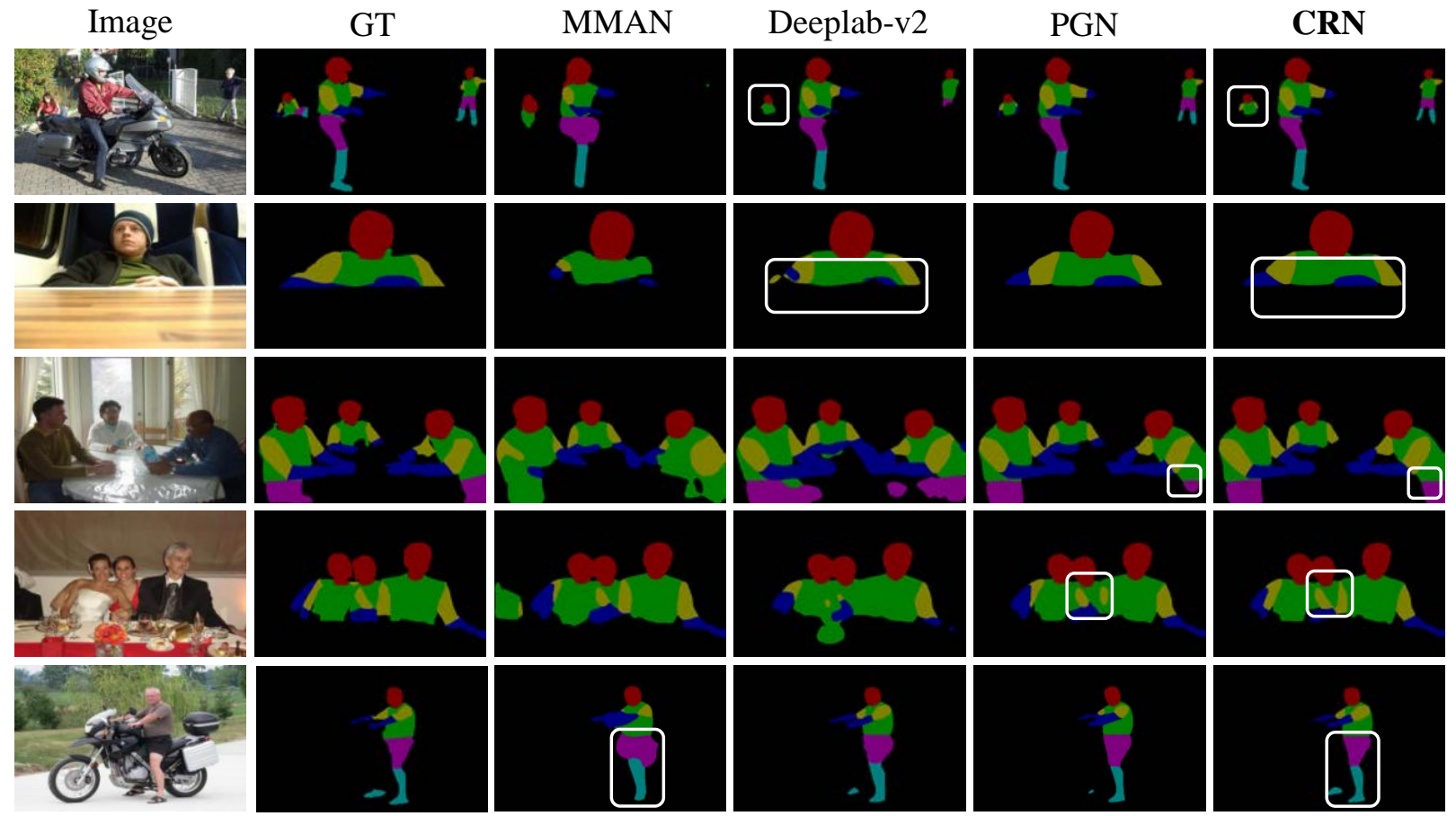

Fig. 3: Some comparison results for human parsing on PPP dataset

(2) Evaluation on CIHP Dataset

Similarly, we perform experiment on the CIHP dataset, and show the comparison results in Table 3. Our method performs better than the current baseline PGN [5] with $1.74 \%$ improvement in terms of mIoU. It indicates that adding cross-refinement branch after ResNet-101 can facilitate identify richer and more precise semantic information, especially for some categories like skirt, hair and face.

Table 3: Comparison results with the current methods on CIHP dataset

\begin{tabular}{|c|c|c|c|}
\hline Method & Mean IoU(\%) & Mean Acc(\%) & Recall(\%) \\
\hline MMAN [13] & 47.31 & 88.02 & 97.69 \\
\hline Deeplab-v2 [2] & 49.18 & 86.52 & 98.84 \\
\hline PGN [5] & 55.89 & 83.33 & 99.00 \\
\hline CRN(Ours) & 57.63 & 88.17 & 99.48 \\
\hline
\end{tabular}

We show the segmentation results on the CIHP dataset in Figure 4, in which the information distribution of each column remains the same as in Figure 3. As shown by the red and green boxes in the Figure 4, the first and the third row show the semantic errors that the semantic label of skirt is recognized as pants or dress. The other rows show incomplete semantic recognition, for example, absence of semantic information of head. It can be clearly seen that our method can solve this two problems very well and generate precise and fine-grained results in multiple-person parsing scenes. 


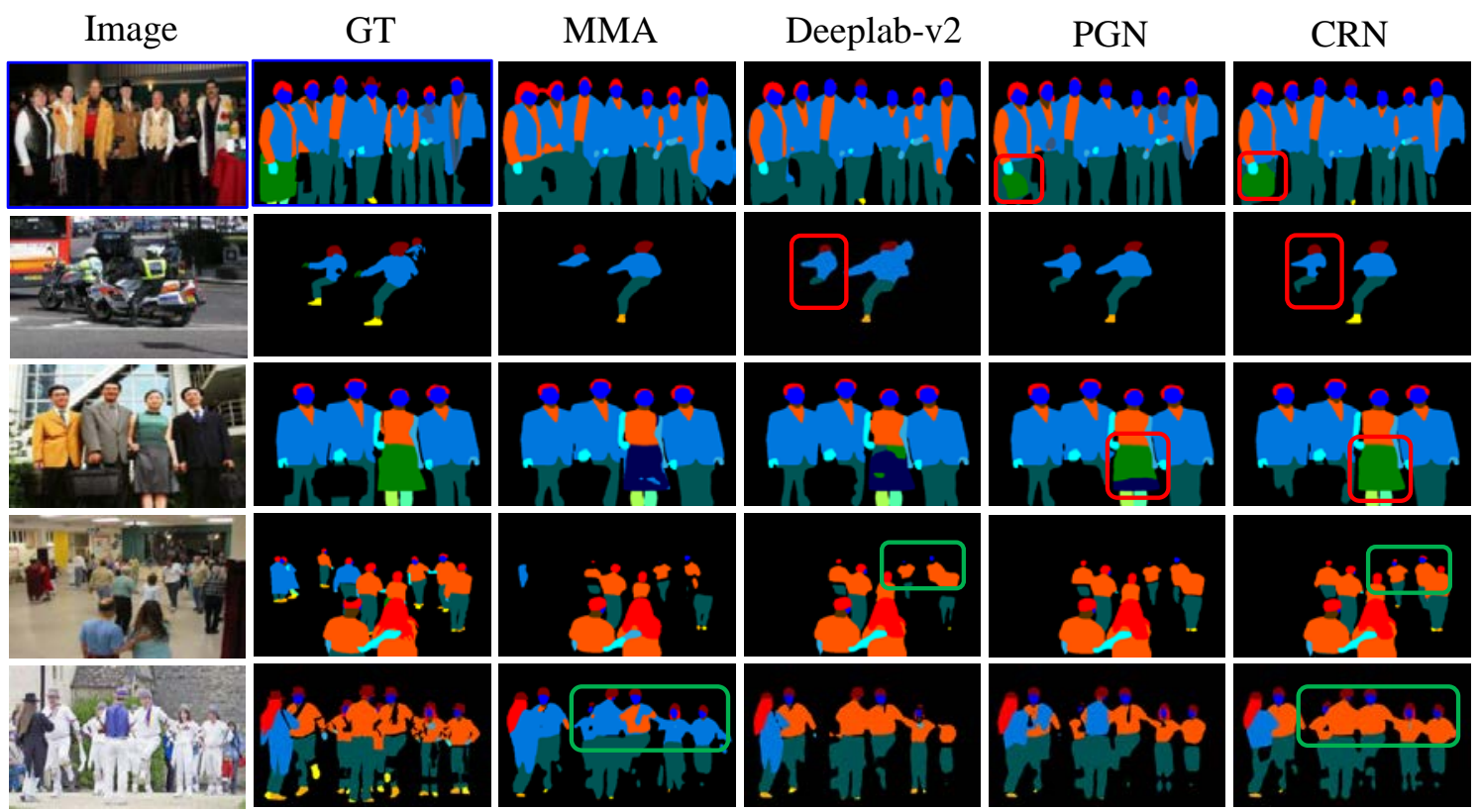

Fig. 4: Comparison results for human parsing on the CIHP dataset

(3) Evaluation on LIP Dataset

Table 4: Comparison with the current methods on LIP dataset

\begin{tabular}{cccc}
\hline Method & Mean IoU(\%) & Mean Acc(\%) & Recall(\%) \\
\hline MMAN [13] & 46.81 & 86.83 & 93.21 \\
Deeplab [2] & 44.80 & 86.33 & 95.72 \\
PGN [5] & 40.21 & 84.45 & 97.07 \\
\hline CRN & $\mathbf{5 2 . 5 7}$ & $\mathbf{8 8 . 1 3}$ & $\mathbf{9 7 . 5 0}$ \\
\hline
\end{tabular}

Furthermore, we evaluate the performance on the largest single-person human parsing dataset LIP. To make a fair comparison with other method, we maintain the entire training epoch unchanged. As presented in Table 4, we can conclude that our method still achieves the best performance in terms of all the three metrics, which illustrates the effectiveness of our CRN. In particular, compared with the current best method MMAN [13], we achieve mIoU improvement by 5.76\%. In addition, As shown in Figure 5 emphasized with the red boxes, our method can segment richer semantic information, such as the skirt in first row, the hand in second row, the head in third row.

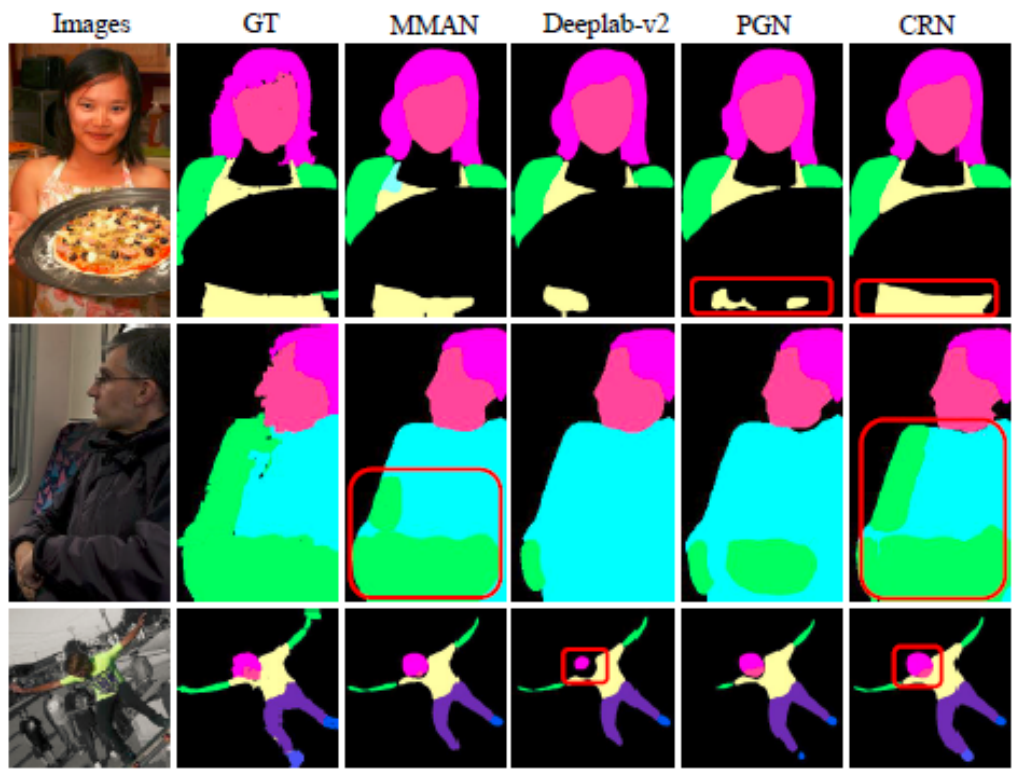

Fig. 5: Some comparison results for human parsing on the LIP dataset 


\section{Conclusion and Future Work}

To solve the problems of inaccuracy of semantic segmentation and lack of human body details in human parsing task, this paper proposed a novel Cross Refinement Network (CRN). Firstly, we use the ResNet-101 as the unified network to train and optimize the shared weights jointly. Secondly, we involve the cross-refinement module consists of the semantic segmentation refinement branch and the edge detection refinement branch, to obtain the complementary contextual information. Finally, we employ a refinement branch to make the two branches further benefit each other. The experimental results on CIHP, PPP and LIP datasets demonstrate that our method improves the performance of human parsing effectively compared to the typical methods.

In the future, we will pay more attentions to the instance-level human parsing with our method and optimize the CRN architecture to generate the better results.

\section{Acknowledgement}

The authors would like to thank the Youth Fund of National Natural Science Foundation of China (No.617021555, No.61906058), Anhui Natural Science Foundation (No.1808085MF176, No. 1908085MF210), the Fundamental Research Funds for the Central Universities of China (Grant No. PA2019GDPK0071), and the National Natural Science Foundation of China (No.61972128), for their supports.

\section{Reference}

[1] J. Long, E. Shelhamer, T. Darrell. Fully Convolutional Networks for Semantic Segmentation. In IEEE Conference on Computer Vision and Pattern Recognition(CVPR), pages 3431-3440, 2015. 1

[2] L. C. Chen, G. Papandreou, I. Kokkinos, K. Murphy, A. L. Yuille. Deeplab: Semantic image segmentation with deep convolutional nets, atrous convolution, and fully connected crfs. In IEEE Transactions on Pattern Analysis and Machine Intelligence, 40(4):834-848, 2017. 1, 6

[3] L. C. Chen, G. Papandreou, F. Schroff, H. Adam. Rethinking Atrous Convolution for Semantic Image Segmentation. arXiv preprint arXiv: 1706.05587, 2017.3, 7

[4] Wang, L., Ji, X., Deng, Q., Jia, M.: Deformable part model based multiple pedestrian detection for video surveillance in crowded scenes. International Conference on Computer Vision Theory \& Application, IEEE, 2014. 2

[5] K. Gong, X. Liang, Y. Li, Y. Chen, M. Yang, L. Lin. Instance-level Human Parsing via Part Grouping Network. arXiv preprint arXiv: 1808.00157, 2018.7,8

[6] N. Souly, C. Spampinato, M. Shah. Semi and Weakly Supervised Semantic Segmentation Using Generative Adversarial Network. arXiv preprint arXiv: 1703.09695, 2017.3

[7] O. Ronneberger, P. Fischer, T. Brox. U-Net: Convolutional Networks for Biomedical Image Segmentation. In Computer Vision and Pattern Recognition (CVPR), 2015.5

[8] V. Badrinarayanan, A. Kendall, R. Cipolla. SegNet: A Deep Convolutional Encoder-Decoder Architecture for Image Segmentation. IEEE Transactions on Pattern Analysis and Machine Intelligence, 2015.11

[9] H. Zhao, J. Shi, X. Qi, X. Wang, J. Jia. Pyramid Scene Parsing Network. In IEEE Conference on Computer Vision and Pattern Recognition (CVPR), pages 2881-2890, 2017. 3, 6, 7

[10] K. Simonyan, A. Zisserman. Very Deep Convolutional Networks for Large-Scale Image Recognition. arXiv preprint arXiv: 1409.1556, 2014. 2

[11] K. He, X. Zhang, S. Ren, J. Sun. Deep Residual Learning for Image Recognition. In Computer Vision and Pattern Recognition (CVPR), pages 770-778, 2016.9

[12] F. Yu, V. Koltun. Multi-Scale Context Aggregation by Dilated Convolutions. arXiv preprint arXiv: 1511.07122, 2015.11

[13] Y. Luo, Z. Zheng, L. Zheng, T. Guan, J. Yu, Y. Yang. Macro-Micro Adversarial Network for Human Parsing. In European Conference on Computer Vision (ECCV), pages 418-434, 2018.7

[14] K. Gong, X. Liang, X. Shen, and L. Lin. Look into person: Self-supervised structure-sensitive learning and a 
new benchmark for human parsing. In IEEE Conference on Computer Vision and Pattern Recognition (CVPR), pages 932-940, 2017. 7

[15] X. Chen, R. Mottaghi, X. Liu, S. Fidler, R. Urtasun, A. Yuille. Detect what you can: Detecting and Representing Objects using Holistic Models and Body Parts. In IEEE Conference on Computer Vision and Pattern Recognition (CVPR), pages 1971-1978, 2014.6

[16] M. Everingham, L. V. Gool, Christopher K. I. Williams, J. M. Winn, and A. Zisserman. The pascal visual object classes (VOC) challenge. IJCV, 88(2):303-338, 2010. 